\title{
Clonage de I'ADNc du récepteur du GHRP: confirmation de la troisième voie contrôlant la sécrétion de l'hormone de croissance (GH)
}

La sécrétion pulsatile d'hormone de croissance par l'antéhypohyse est contrôlée par deux peptides hypothalamiques antagonistes: la somatostatine, qui l'inhibe, et la GHRH (GHreleasing hormone) qui la stimule. Ces deux peptides ont été purifiés et caractérisés ces vingt-cinq dernières années et leurs mécanismes d'action en grande partie élucidés. Les $\mathrm{ADNc}$ codant pour leurs récepteurs ont, de même, plus récemment été clonés; ces récepteurs appartiennent à la famille des récepteurs à sept domaines transmembranaires couplés aux protéines G. La somatostatine inhibe la formation d'AMP cyclique, alors que la GHRH la stimule. La possibilité d'un troisième intervenant dans la régulation complexe de la sécrétion circadienne de GH a été envisagée lors de la mise en évidence, in vitro et in vivo, de l'action stimulatrice sur la sécrétion de GH d'un hexapeptide de synthèse dérivé des enképhalines, le GHRP-6 (growth hormone-releasing peptide). Le GHRP-6, comme un autre peptide l'hexareline, ou des analogues non peptidiques développés par la suite comme le L-692-429 ou le MK-0677, stimule et amplifie la sécrétion de GH par un récepteur différent de celui de la GHRH et non couplé à l'adénylyl cyclase. Le GHRP et ses analogues amplifient aussi la sécrétion pulsatile de $\mathrm{GH}$ en potentialisant l'action de la GHRH. On envisage l'utilisation de ces analogues, dont certains ont une bonne biodisponibilité orale, dans le traitement de retards de croissance par déficit en GH; c'est dire l'intérêt que l'on portait à la caractérisation du récepteur du GHRP. Son étude sur des membranes hypophysaires indiquait qu'il était sans doute couplé aux protéines $G$ [1]; son activation entraîne une dépolarisation avec inhibition des canaux potassiques, augmentation des taux intracellulaires de calcium, des inositol triphosphates, et de l'activité de la protéine kinase $\mathrm{C}$ (PKC). Le GHRP-6 et ses analogues non peptidiques agissent aussi bien au niveau hypophysaire qu'au niveau hypothalamique (en particulier par stimulation de la sécrétion de la GHRH par les neurones du noyau arqué de l'hypothalamus médiobasal). En outre, un couplage anormal du récepteur du GHRP semble présent dans les adénomes hypophysaires à GH porteurs de l'oncogène Gsp (mutation activatrice de la sousunité $\alpha \mathrm{S}$ de la protéine Gs $\left(\mathrm{m} / \mathrm{s} n^{\circ} 12\right.$, vol. 9, p. 1421), conduisant à une activation constitutionnelle de l'adénylyl cyclase). Dans ce sous-groupe d'adénome, le GHRP est capable de stimuler la formation d'AMP cyclique (à la différence de l'absence de réponse observée sur l'hypophyse normale ou les adénomes sans mutation), évoquant la possibilité d'une interaction entre la voie de la PKC et celle de l'AMP cyclique dans ces tumeurs [2]. L'équipe de LHT Van der Ploeg (Merck Research Lab, Rahway, NJ, USA) a cloné les ADNc des récepteurs humain et porcin du GHRP [3]. Ayant réalisé l'expression fonc- tionnelle d'ARN poly (A) ${ }^{+}$d'hypophyse de porc dans des œufs de xénope co-injectés avec l'ARN complémentaire de la sous-unité $\alpha$ de la protéine G11 (activant la phospholipase C), ils mirent en évidence une augmentation du calcium intracellulaire en réponse au GHRP6 et à son analogue non peptidique, le MK0677. On doit noter que l'injection des autres sous-unités $\alpha$ des protéines $G\left(\alpha_{q}, \alpha_{16}, \alpha_{13}, \alpha_{i 1}, \alpha_{i 3}, \alpha_{o}\right)$ ne permettait pas d'obtenir de réponse au GHRP. Cette approche permit le clonage des ADNc codant pour les récepteurs porcin et humain du GHRP (Ia). Il s'agit d'une protéine de 366 acides aminés présentant les caractéristiques des récepteurs à sept domaines transmembranaires. Un deuxième $\mathrm{ADNc}$ (Ib) a aussi été cloné dans les mêmes espèces; la protéine déduite ne comporterait que cinq domaines transmembranaires et, à la différence du type Ia, ce récepteur n'est pas fonctionnel dans les œufs de xénopes. Les deux $\mathrm{ADNc}$ sont très probablement les produits de l'épissage alternatif du même gène. Une analogie très forte existe entre les récepteurs Ia des deux espèces, puisque $93 \%$ des acides aminés les composant sont identiques. L'analyse du gène du récepteur du GHRP est en faveur d'un gène unique très conservé chez les différents vertébrés étudiés (gènes humain, porcin, bovin, murin, du chimpanzé et du rat). En outre, les récepteurs Ia et Ib semblent être des protéines originales dans la famille 
des récepteurs couplés aux protéines $\mathrm{G}$, puisque les séquences des récepteurs les plus proches (TRH et neurotensine) ne comportent que 29 à $35 \%$ d'acides aminés identiques. La liaison du GHRP-6 et de ses analogues au récepteur Ia synthétisé dans des cellules COS-7 est spécifique, saturable et de forte affinité, et est bien corrélée à l'activité biologique observée in vivo. L'étude de l'expression tissulaire du récepteur chez l'homme et le primate montre qu'il est spécifique du système hypothalamo-hypophysaire. Il est synthétisé en quantité dans l'hypophyse et dans l'hypothalamus médio-basal à proximité de l'éminence médiane (noyau arqué ou infundibulaire). Ce récepteur présent dans l'hypothala- mus médio-basal pourrait relayer le contrôle de l'activité sécrétoire des neurones à GHRH, et constituer un élément important dans la pulsatilité de la sécrétion de la GH.

Le clonage de l'ADNc du récepteur du GHRP a permis de démontrer clairement l'existence d'un troisième partenaire participant au contrôle de l'activité de l'axe somatotrope à coté de la somatostatine et de la GHRH. L'existence de ce récepteur n'était, jusqu'à ce jour, que postulée à partir d'observations faites avec des ligands synthétiques. Le ligand endogène du récepteur du GHRP reste encore à identifier. Son étude promet certainement de nouvelles perspectives de compréhension du phénomène complexe, et qui n'est que partiellement élucidé, des mécanismes contrôlant la pulsatilité de la sécrétion de GH.

J.B.

1. Pong SS, Chaung LYP, Dean DC, Nargund RP, Patchett AA, Smith RG. Identification of a new Gprotein linked receptor for growth hormone secretagogues. Mol Endocrinol 1996; 10 : 57-61. 2. Adams EF, Lei T, Buchfelder M, Bowers CY, Fahlbusch R. Protein Kinase C-dependent growth hormone releasing peptides stimulate cyclic adenosine 3',5'-Monophosphate production by human pituitary somatotropines expressing gsp oncogenes: evidence for cross-talk between transduction pathways. Mol Endocrinol 1996; 10: 432-8. 3. Howard AD, Feighner SD, Cully DF, et al. A receptor in pituitary and hypothalamus that functions in growth hormone release. Science 1996; 273: $974-7$

\section{a BRÈVES}

Des précisions sur la transmission intracellulaire des signaux inflammatoires. Parallèlement à la voie de transmission du signal aboutissant aux MAP-kinases de type ERK (extracellular signal-regulated kinases), un signal aboutit à l'activation de la kinase JNK (c-Jun amino-terminal kinase), également appelée SAPK (stress-activated protein kinase). L'activation de la JNK/SAPK aboutit à la phosphorylation de c-Jun et à l'augmentation de l'activité du facteur de transcription AP1. Ce signal inflammatoire est engendré par des récepteurs couplés aux grandes protéines $\mathrm{G}$, mais aussi par le choc osmotique, les rayons ultraviolets et des cytokines de la famille du TNF. Certains des partenaires de la cascade aboutissant à l'activation de JNK/SAPK ont été identifiés: les petites protéines $\mathrm{G}$ de la famille Rho, Rac, CDC42Hs. Des kinases capables de phosphoryler la JNK/ SAPK (SAPKK ou MEK; $m / s n^{\circ}$ 3, vol. 11, p. 467) ont également été caractérisées. L'équipe de Schlessinger, de New York (USA) démontre maintenant l'intervention d'une tyrosine kinase Pyk [1]. Pyk2 ressemble à la kinase FAK (focal adhesion kinase) $\left(\mathrm{m} / \mathrm{s} n^{\circ} 3\right.$, vol. 11, p. 491); elle est activée par le
TNF $\alpha$, par les rayons ultraviolets et par les modifications de l'osmolarité. Sa surexpression conduit à l'activation de la JNK/SAPK alors qu'un mutant transdominant négatif diminue l'activation de cette kinase par les ultraviolets et le choc osmotique. Pyk2 est, par conséquent, une tyrosine kinase sensible au stress, impliquée dans l'activation du signal inflammatoire.

[1. Tokiwa G, et al. Science 1996 ; 273: 792-4.]

L'inversion thérapeutique des lésions dégénératives du diabète est-elle possible? L'un des mécanismes des complications dégénératives liées au diabète, passant par l'hyperglycémie chronique, est la glycosylation (aussi appelée glycation) anormale des protéines aboutissant, pour certaines d'entre elles à durée de vie prolongée, à la formation de produits stables formés de molécules glycosylées liées entre elles de manière covalente, dénommés AGE (advanced-glycation end-products) $\left(\mathrm{m} / \mathrm{s} n^{\circ} 11\right.$, vol. 10, p. 1165). La formation de tels AGE dans les tissus conjonctifs et au niveau de la matrice extracellulaire pourrait être l'une des bases lésionnelles de beaucoup des complications dégénératives du diabète, du vieillissement, et même de la maladie d'Alzheimer. Le mode exact de formation des lésions covalentes entre les protéines glycosylées n'est pas connu mais on pense que des intermédiaires di-carbonyles (ou dicétones) pourraient être impliqués. Une équipe américaine de chercheurs académiques (Minneapolis, $\mathrm{MN}$ ) et industriels (Ramsey, NJ) vient de montrer qu'un produit, le bromure de N-phénacylthiozolium (PTD), était capable de cliver les liaisons covalentes des AGE, in vitro et in vivo. Naturellement, on ne sait rien de l'effet thérapeutique d'une telle dégradation des AGE ni de la tolérance à court et long terme du PTD. Cependant, la plupart d'entre nous avons le sentiment que les complications dégénératives et les changements liés au vieillissement aboutissent à des lésions irréversibles. Il est donc intéressant de montrer que cela n'est pas obligatoirement une fatalité.

[1. Vasan S, et al. Nature 1996; 382 : 275-8.] 